\title{
Ultrasound Elastography Used for Preventive Non-Invasive Screening in Early Detection of Liver Fibrosis
}

\author{
Florian Bert ${ }^{\mathrm{a}, \mathrm{c}}$, Jona T. Stahmeyer ${ }^{\mathrm{b}}$, Siegbert Rossol ${ }^{\mathrm{a}}$
}

\begin{abstract}
Background: Early discovery of liver fibrosis is becoming more popular because of enhanced incidence of hepatocellular carcinoma. Ultrasound-based liver elastography is a method used to approve suspected liver fibrosis or cirrhosis. We assessed the clinical usefulness of acoustic radiation force impulse shear wave elasticity imaging (ARFI-SWEI) as a preventive screening method to uncover fibrosis.

Methods: We screened 382 patients by native routine sonography for abnormal liver results and divided them into six groups: group 1: normal liver, groups 2-4: fatty liver grade I-III, group 5: liver cirrhosis, and group 6: inhomogenic liver tissue. Then ARFI-SWEI was performed and the results were compared with published shear wave velocity cut-off values that were predictive of each fibrosis stage (F04). A control group consisted of 20 healthy volunteers.
\end{abstract}

Results: The part of liver fibrosis $\geq$ F2 was in groups 1-4: 20-32\%, group 5: $100 \%$, and group 6: $91 \%$. Main causes for fibrosis stage $\geq$ F2 were (non)-alcoholic steatohepatitis, chronic viral or autoimmune hepatitis and chronic heart failure.

Conclusions: Screening of the liver tissue in b-mode ultrasound can underestimate possible liver fibrosis; by using ARFI-SWEI, liver fibrosis can be uncovered early. It is a suitable preventive method comparable to colonoscopy for colon cancer.

Keywords: Ultrasound; Liver fibrosis; Elastography; ARFI

\section{Introduction}

In recent years, the early discovery of progressive liver failure and liver fibrosis is becoming more popular because of enhanced incidence of hepatocellular carcinoma (HCC). HCC

Manuscript accepted for publication July 01, 2016

aDepartment of Internal Medicine, Krankenhaus Nordwest, Steinbacher Hohl 2-26, 60488 Frankfurt/Main, Germany

'Institute of Epidemiology, Social Medicine and Health System Research, Hannover Medical School, Carl-Neuberg-Str. 1, 30625 Hannover, Germany

${ }^{\mathrm{c} C o r r e s p o n d i n g ~ A u t h o r: ~ F l o r i a n ~ B e r t, ~ D e p a r t m e n t ~ o f ~ I n t e r n a l ~ M e d i c i n e, ~}$ Krankenhaus Nordwest, Steinbacher Hohl 2-26, 60488 Frankfurt/Main, Germany. Email: florianbert@freenet.de

doi: http://dx.doi.org/10.14740/jocmr2625w is the third leading cause of cancer-related death and the fifth most common malignancy in men and the seventh in women worldwide $[1,2]$. Nearly $95 \%$ of all HCCs are based on liver cirrhosis (LC) with an incidence up to $6 \%$ per year [3]. LC is the consequence of chronic liver disease (CLD). Non-alcoholic or alcoholic fatty liver disease ((N)AFLD) and chronic viral hepatitis $\mathrm{B}$ and $\mathrm{C}(\mathrm{HBV} / \mathrm{HCV})$ are leading CLDs in the western world [4]. They induce an inflammatory response of the liver tissue with activation of immune cells, proliferation and transdifferentiation of mesenchymal cells and following progress to liver fibrosis up to LC [5].

Epidemiologic data from Germany estimate that nearly 4 - 5 million people suffer from CLD [6]. Mortality analysis shows that CLD as a cause of death ranges on place 5 in Germany and the risk to develop LC is up to 5\% [5]. Data analysis from the US Department of Health and Human Services revealed that CLD ranges on place 12 of 15 leading causes of death in 2013 [7].

Often patients do not know about their possible liver failure because typical symptoms like fatigue or jaundice are missing until the late stage of the disease [8]. Today there exist successful therapeutic options for chronic $\mathrm{HBV} / \mathrm{HCV}$ and the risk of following LC and/or HCC decreases. But steatosis hepatis and steatohepatitis are more and more problem for people of the industrialized countries. Less sporting activity, excess of calories and/or alcohol are the main reasons for developing overweight, fatty liver and/or steatohepatitis with elevated risk of LC and HCC. The obesity prevalence ranges from $2.3 \%$ to $12 \%$, and overweight is $28.8 \%$, mostly affecting females [9]. Nowadays (N)AFLD-associated LC is the second leading cause for liver transplant in the USA and will overtake the chronic HCV in the next years [10].

The life expectancy and prognosis of patients with LC are significantly lower with 1-year mortality of $40 \%$ [11]. Mortality and morbidity rates increase exponentially once cirrhosis develops; a systematic review of 118 studies involving 23,797 patients with LC by D'Amico et al showed that the overall survival was only $64 \%$ over a median follow-up of 31 months [12].

To prevent LC, the aim is to uncover early stages of a liver fibrosis and corresponding CLD [13]. In the past, the diagnosis and follow-up of progressive liver fibrosis are based on histological examination using liver biopsy. Although biopsy is still considered the "gold standard" for histological evaluation, it is well known that this procedure has several limitations (i.e., sampling errors, bleeding complications) and poor tolerance as a stressful medical procedure for many patients $[13,14]$.

Thus, in recent years, interest increased in identifying and describing liver fibrosis by using non-invasive technical methods [14]. Acoustic radiation force impulse shear wave elastic- 


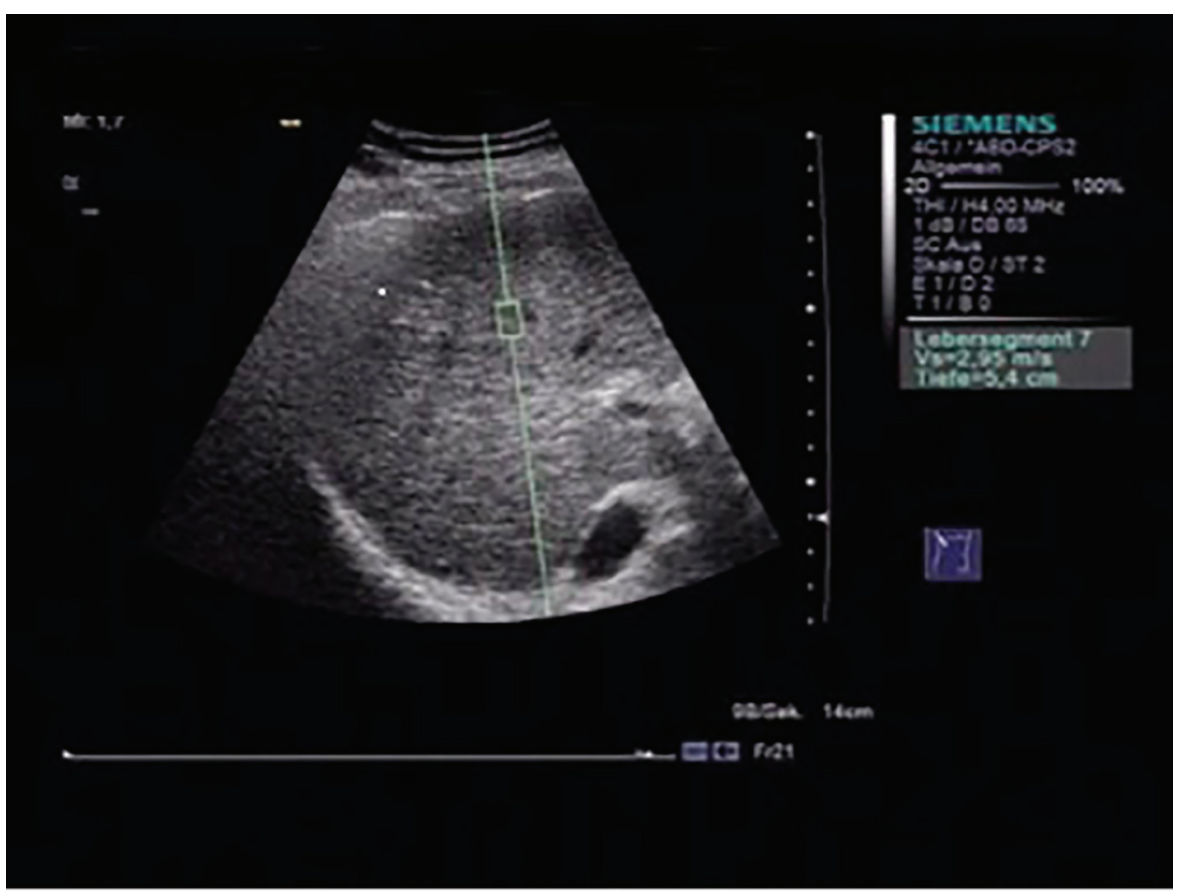

Figure 1. Acoustic radiation force impulse (ARFI) measurement, with the flexible metering box ("measurement box" as size of region of interest $(\mathrm{ROI}), 10 \mathrm{~mm}$ long, $5 \mathrm{~mm}$ wide) is possible to localize a region of the liver for elastographic measurement at variable depths.

ity imaging (ARFI-SWEI) is a novel ultrasound method for liver fibrosis assessment [15-17]. This dynamic method uses acoustic radiation force to produce an acoustic "push" pulse that generates shear waves, which propagate into the tissue; their speed reflects the underlying tissue stiffness and severity of liver fibrosis [18]. This method is comfortable for the patient and examiner. It can be used for assessing different degrees of liver stiffness (fibrosis stages) and correlated to the b-mode aspect of the liver in the same session [19].

Recent studies verified ARFI-SWEI as a powerful noninvasive method in predicting fibrosis $\geq$ F2 with high diagnostic accuracy and validity [18]. Because of its accuracy and easy handling, point of interest is to use this method for early discovering liver fibrosis independent of knowledge of a causal CLD. For this reason, the purpose of this study was to investigate the clinical usefulness of ARFI-SWEI screening to uncover possible liver fibrosis in patients of a large teaching hospital during routine ultrasound of the abdomen.

\section{Materials and Methods}

\section{Patients}

In this cross-section study, over a period of 6 weeks, we screened 403 patients of nine departments (departments of surgery (visceral, thoracic, and orthopedic), gynecology, urology, neurology, oncology, radio-oncology, and internal medicine) of the Krankenhaus Nordwest in Frankfurt/Main for striking findings of liver by using routine ultrasound and additional liver elastography (ARFI-SWEI).

The clinical cause for patient hospitalization was of no interest for the study. To avoid a bias, each patient taking part in the study should get any kind of ultrasound examination, independent of its indication and region to be investigated (e.g. thyroid gland, abdomen, vessels), and was screened with abdominal ultrasound and ARFI-SWEI.

Additional blood samples of the participants were taken to determine the platelet count and the alanine aminotransferase (ALT) looking for signs of CLD in context of liver fibrosis. The correlation of severity of liver damage by histology was not the aim of this study, neither the estimation of the prognosis of the surveillance.

The study was conducted in compliance with the Declaration of Helsinki. The institutional review board approved the study and the patients were informed of the study procedure.

Twenty-one patients $(4.5 \%)$ of 403 enrolled patients were not suitable for ARFI-SWEI measurement, because of low intercostal access $(\mathrm{N}=4)$, high body mass index $(\mathrm{BMI}>30 \mathrm{~kg} /$ $\left.\mathrm{m}^{2}, \mathrm{~N}=10\right)$ or missing cooperation $(\mathrm{N}=7)$ during the procedure. Thus, a total of 382 patients were included in the study.

An additional control group consisted of 20 healthy volunteers (10 male and 10 female), with a median age of $50 \pm 14$ years without known history of liver damage.

\section{Native ultrasound scan and ARFI-SWEI}

Two physicians performed ultrasound scan and elastographic measurements independently with an ultrasound unit by Siemens AG (Germany), Acuson S 2000, which included the Vir- 
tual Touch ${ }^{\mathrm{TM}}$ Tissue Quantification Software for ARFI-SWEI technology.

In the first step, we evaluated the liver tissue of the participants by b-mode native ultrasound. In case of fatty liver, the degree (grade 1-3) was defined according to the method by Saverymuttu (1986); the criterion for fatty liver was hyperechogenic liver tissue with fine, tightly packed echoes; as a reference for comparing normal liver tissue, we used the tissue of the kidney [20]. The ultrasound unit contains tissue harmonic imaging (THI), which resolves the problems related to ultrasound in technically difficult patients by providing a marked improvement in image quality. We used an Acuson S2000 4C1 curved transducer with a frequency of $4.5 \mathrm{MHz}$ and mechanical index of $1.1-1.2$.

\section{ARFI-SWEI technique}

ARFI-SWEI technology is a real-time dual display imaging mode and allows a quantitative assessment of tissue stiffness, through measurement of shear wave velocity (SWV). Shear waves are generated by displacement of tissue and attenuate approximately 10,000 times more rapidly than conventional ultrasound waves.

With a flexible metering box ("measurement box" as size of region of interest (ROI), $10 \mathrm{~mm}$ long, $5 \mathrm{~mm}$ wide), it is possible to localize a region of the liver for elastographic measurement at variable depths. An acoustic push pulse transmitted by the transducer toward the tissue induces an elastic shear wave that propagates through the tissue. The propagation of the shear wave is followed by detection pulses that are used to measure the velocity of shear wave propagation, which is directly related to tissue stiffness, and is quantified in meters
Table 1. Results of Native Ultrasound Scan of the Liver

\begin{tabular}{ll}
\hline $\begin{array}{l}\text { Native ultrasound } \\
\text { (US) results }\end{array}$ & Group (groups 1-6: $\mathbf{N}=\mathbf{3 8 2}$ ) \\
\hline Normal native US & Controls $(\mathrm{N}=20)$; group 1 $(\mathrm{N}=131)$ \\
\hline Fatty liver grade I & Group $2(\mathrm{~N}=70)$ \\
\hline Fatty liver grade II & Group $3(\mathrm{~N}=34)$ \\
Fatty liver grade III & Group $4(\mathrm{~N}=45)$ \\
Cirrhotic tissue & Group $5(\mathrm{~N}=43)$ \\
\hline Inhomogenic tissue & Group 6 $(\mathrm{N}=59)$ \\
\hline
\end{tabular}

per second (m/s) [13, 16] (Fig. 1).

To optimize the results, we standardized our ARFI-SWEI measurement. We did the examination in the morning and the patients were without food intake and fasting $>3 \mathrm{~h}$. The patient was arranged in dorsal decubitus position, with the right arm under his head (maximum abduction). We made ARFI-SWEI measurement at the level of the right liver lobe segment VII/ VIII at a depth between 3.5 and $8.0 \mathrm{~cm}$, while the patients were asked to stop breathing for a moment [17].

We performed 10 valid measurements in every patient, and the ratio between validated and total measurements as a median value was calculated.

Only procedures with a success rate of at least $60 \%$, and with the interquartile range of all validated measurements $<30 \%$ of the median value, were considered reliable [18]. We compared the results of ARFI-SWEI measurements with published SWV cut-off values that were predictive for each fibrosis stage correlated to the histological classification of METAVIR (F0-4): F0-1 $<1.24 \mathrm{~m} / \mathrm{s}, \mathrm{F} 2 \geq 1.25 \mathrm{~m} / \mathrm{s}, \mathrm{F} 3 \geq 1.60 \mathrm{~m} / \mathrm{s}$ and F4 $\geq 2.00 \mathrm{~m} / \mathrm{s}[21$, 22]. A distinguishing between $\mathrm{F} 0$ and $\mathrm{F} 1$ and conclusions about

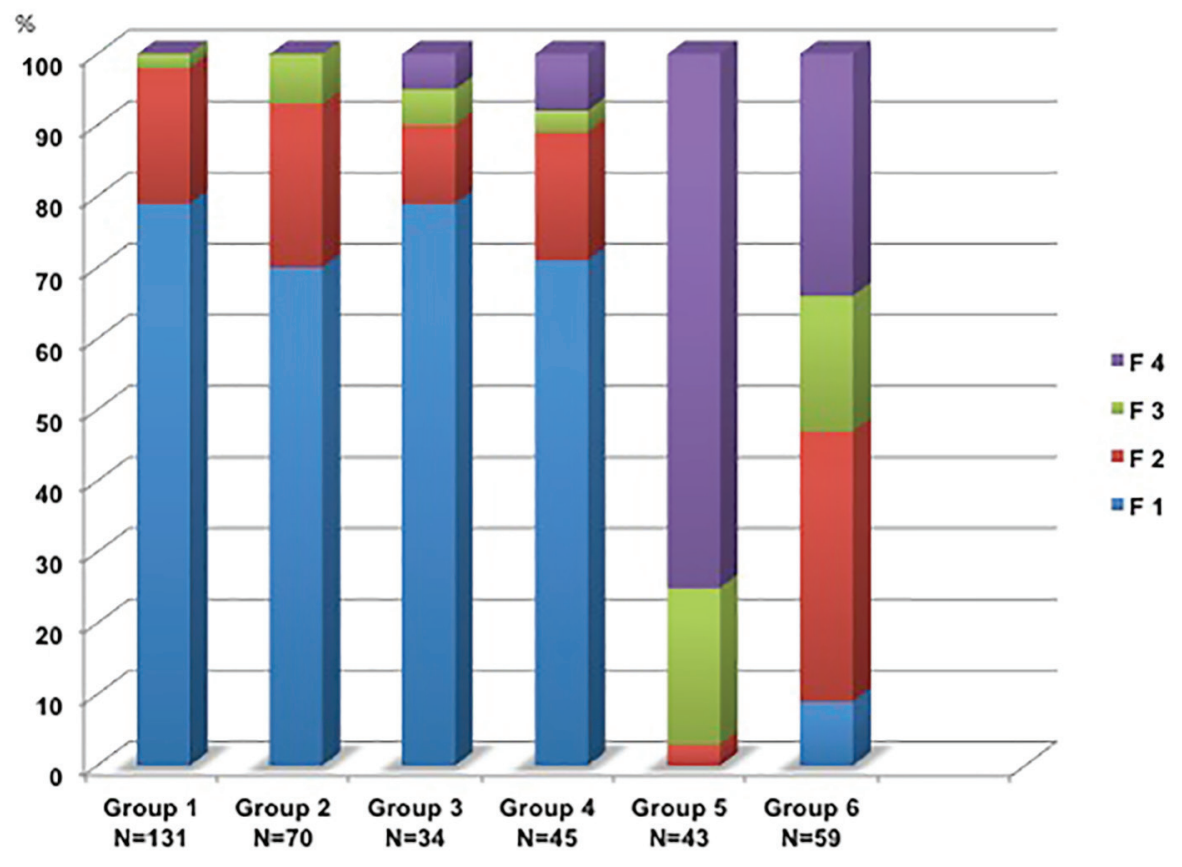

Figure 2. Fibrosis stages F1*-F4 in groups 1-6. (*Stage F1 includes although F0). 


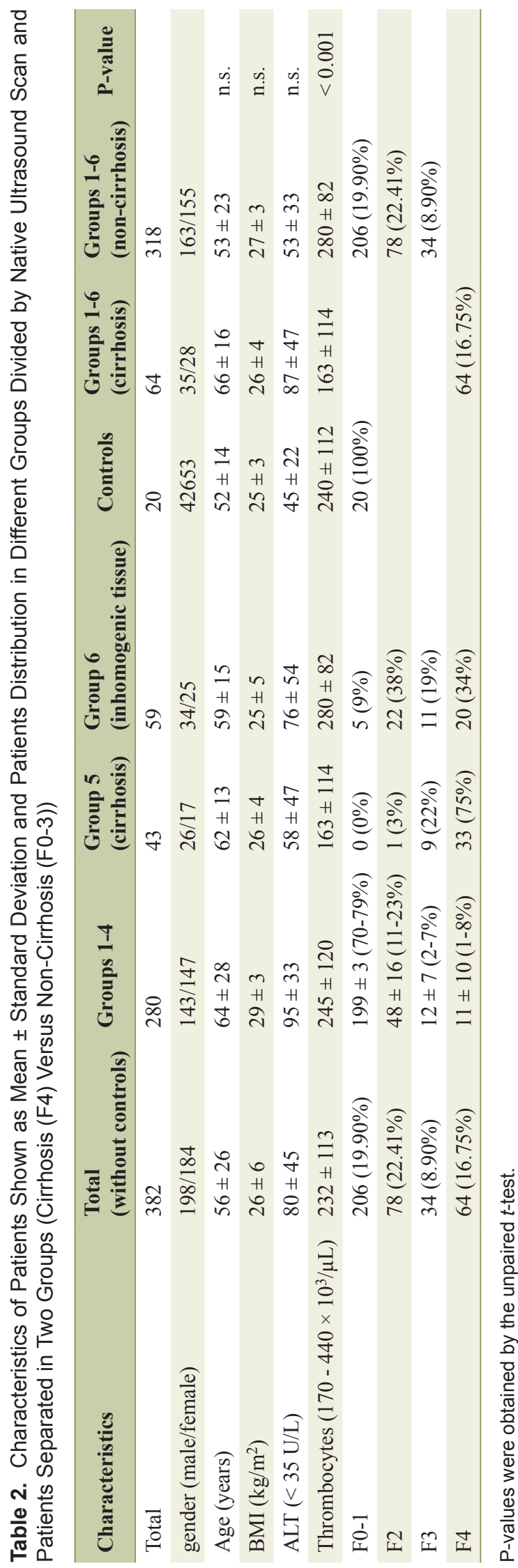

the activity of (necro-)inflammation in the liver tissue is impossible for ARFI-SWEI and can be estimated by histology only.

In case of elevated SWV with $\geq$ F2 (comparable to METAVIR: portal fibrosis with few septa), the patient history was scrutinized for further hints of CLD.

\section{Statistical analysis}

The results and measurements were presented as the mean \pm standard deviation (SD) or percentages, as appropriate. P-values were obtained by the unpaired $t$-test. For statistical analysis, we used Microsoft Excel.

\section{Results}

A total number of 382 patients were included in the study. According to the results of native ultrasound scan, the participants were divided into seven different groups (Table 1): controls, group 1: normal tissue, group 2: steatosis grade 1, group 3: steatosis grade 2, group 4: steatosis grade 3 , group 5: cirrhotic tissue, and group 6: inhomogenic tissue.

The ARFI results are shown in Figure 2.

Groups 1-4 showed nearly the same distribution of fibrosis grading, independent of the steatosis grade: F0-1 68-79\%, F2 $11-19 \%, F 32-7 \%$ and F4 0-8\%. In contrast, the part of F4 exceeded over $75 \%$ in group 5 corresponding to the native bmode. Group 6 showed no signs of fatty liver or cirrhosis. The liver tissue b-mode scan was described as inhomogenic and over $91 \%$ of the participants in this group had SWV values indicating $\geq F 2$. The additional control group $(\mathrm{N}=20)$ had no signs of fatty liver and ARFI-SWEI SWVs were between 0.70 and $1.00 \mathrm{~m} / \mathrm{s}$ (median: $0.85 \mathrm{~m} / \mathrm{s}$ ) in the normal range.

The BMI was comparable in all other groups except controls, with highest values for groups 2-4, corresponding to fatty liver in the b-mode scan. The distribution of the participant gender in total and more obvious in subgroups 5 and 6 shows an overlap of male versus female.

Analysis of ALT and thrombocyte count shows elevated values for ALT in all groups in context with elevated ARFI-SWEI SWV-values $\geq F 2$, but also in some patients with F0-1. In total there were no correlation found (no significance). Thrombocyte counts were significant reduced in patients with $\mathrm{F} 4$ only (Table 2).

Looking for reasons of elevated SWV $(\geq F 2)$ in each group, we screened the history of those patients for possible liver failure; this added up to five different main causes: alcoholic (ASH, $\mathrm{N}=43$ ) and non-alcoholic steatohepatitis (NASH, $\mathrm{N}=47$ ), viral hepatitis $\mathrm{B} / \mathrm{C}(\mathrm{N}=12)$, chronic heart failure ( $\mathrm{CHF}, \mathrm{N}=$ 20 ), and autoimmune hepatitis (AIH)/primary biliary cirrhosis $(\mathrm{PBC}) /$ primary sclerosing cholangitis $(\mathrm{PSC})(\mathrm{N}=10)$ (Table 3).

\section{Discussion}

Liver fibrosis is a major public health problem with risk of developing LC and HCC. In CLD, the evaluation of liver fibrosis is essential. Recent studies show that ARFI-SWEI is a reli- 
Table 3. Distribution of Patients With Elevated SWV (F2-4): Main Causes

\begin{tabular}{lllllll} 
& Group 1 $(\mathbf{N}=\mathbf{3 3})$ & Group 2 $(\mathbf{N}=\mathbf{2 2})$ & Group 3 $(\mathbf{N}=\mathbf{9})$ & Group 4 (N= 13) & Group 5 (N=43) & Group 6 (N= 53) \\
\hline ASH & 0 & 8 & 2 & 5 & 10 & 18 \\
Hepatitis B/C & 3 & 0 & 1 & 2 & 3 & 3 \\
Heart failure & 7 & 3 & 1 & 1 & 3 & 5 \\
NASH & 0 & 9 & 4 & 0 & 4 & 16 \\
AIH/PBC/PSC & 3 & 0 & 0 & 2 & 7 & 3 \\
Unknown & 20 & 2 & 1 & 3 & 9 \\
\hline
\end{tabular}

able method to evaluate liver stiffness. In our study, we proved ultrasonography-based ARFI-SWEI elastography for its clinical usefulness as a screening method to uncover liver fibrosis.

The accuracy of ARFI-SWEI is well tested and it is reliable in prediction of fibrosis in early stages $(\geq F 2)[13,19]$. The sensitivity and specificity of the ARFI-SWEI method is ascertained of $83-88 \%$ and $89-90 \%$ and its accuracy, expressed as area under the curve receiver-operating characteristic (AUROC), is near $1[13,19,23]$. Because of its reliability, we supported that elevated SWV values indicate a possible liver failure.

Our procedure of ARFI-SWEI was standardized to ensure that the results of the patients were comparable. ARFI-SWEI measurement failed in $4.96 \%$ (21 of 403 patients) because of low intercostal access, heightened BMI or uncooperative patient cooperation. This is comparable to literature data (2.4$9.4 \%$ ) [24].

Ultrasound is the most sufficient technique to detect steatosis of the liver and allows morphologic description of the liver tissue with its potential content of fat. In our study, we separated the participants with the native ultrasound in three different groups (groups 2-4) according to the steatosis degree. Nearly $15-22 \%$ of these patients had steatosis and elevated SWV corresponding to $\geq \mathrm{F} 2$; the grade of steatosis was independent of liver fibrosis stage because the percentage of F2-4 was more elevated in group 2 than in groups 3 and 4 (32\% vs. $23 \%$ vs. 30). Motosugi et al (2011) found out in 200 individuals that steatosis alone without histology assured inflammation shows normal SWV values: the SWV results of the fatty liver group were similar to those of the normal liver group (1.03 \pm 0.12 vs. $1.02 \pm 0.12 \mathrm{~m} / \mathrm{s}(\mathrm{P}<0.0001))$ and the authors concluded that steatosis alone does not affect the liver stiffness measurement [25]. Fibrosis $\geq$ F2 in context with steatosis is a possible hint for steatohepatitis which can lead to liver cirrhosis. In groups 2-4, the leading cause for steatosis seemed to be $(\mathrm{N}) \mathrm{ASH}$. Whereas patients of group 1 (33/131) show elevated SWV ( $\geq$ F2) caused by heart failure, viral hepatitis or autoimmune hepatitis, but in nearly $60 \%$ (20/33) of the cases, the cause of SWV elevation was unclear and further diagnostic was recommended. For group 5, the main reasons for liver cirrhosis were ASH and NASH (>50\%). Even though the bmode scan gave no hints of fatty liver, the fibrotic/cirrhotic transformation was based on (N)ASH in the past. This was also supposed for group 6.

ARFI-SWEI is a reliable tool to determine the degree of liver fibrosis in context of liver failure and CLD. Serological marker and b-mode ultrasound alone can underestimate the progressive liver failure with fibrosis. Only the thrombocyte count in patients with LC showed significant correlation. (N) ASH seemed to be the main causes for liver fibrosis in our study. Both are frequent CLDs in the industrialized world. Their incidences will raise in the future, especially NASH. ARFI-SWEI is useful and comfortable for detecting early fibrotic alterations of the liver tissue. Consecutively protective action can be implemented.

\section{Conclusion}

The results of our study show that in cases of normal native ultrasound scan, liver damage can be underestimated; by using ARFI-SWEI as an additional method, a tissue damage of the liver can be uncovered for further diagnostic evaluation. The method is comfortable for the physician and the patient; it is further useful in cases of known liver damage for a longitudinal follow-up. Liver biopsy with its complications (i.e. pain, bleeding) can be avoided. The ARFI-SWEI system integrated in an ultrasound device has a good accuracy and the reproducibility is high. The evaluation of liver stiffness corresponding to native ultrasound scan is fast. This procedure is suitable for preventive measure to uncover CLD comparable to preventive colonoscopy for colon cancer. Further socioeconomic studies in view of the preventive effect and costs were necessary.

Our results show that ARFI-SWEI is an adequate method in terms of fast examination of the liver in routine ultrasound. In particular fibrosis stage F2 and higher are of interest and for this ARFI-SWEI has high sensitivity and specificity.

\section{Competing Interests}

The authors declare that they have no competing interests.

\section{Grant Support}

None.

\section{Financial Disclosure}

All authors have no financial disclosure to report. 


\section{References}

1. Aghemo A, Colombo M. Hepatocellular carcinoma in chronic hepatitis C: from bench to bedside. Semin Immunopathol. 2013;35(1):111-120.

2. Paradis V. Histopathology of hepatocellular carcinoma. Recent Results Cancer Res. 2013;190:21-32.

3. Lencioni R, Kudo M, Ye SL, Bronowicki JP, Chen XP, Dagher L, Furuse J, et al. First interim analysis of the GIDEON (Global Investigation of therapeutic decisions in hepatocellular carcinoma and of its treatment with sorafeNib) non-interventional study. Int J Clin Pract. 2012;66(7):675-683.

4. Schutte K, Kipper M, Kahl S, Bornschein J, Gotze T, Adolf D, Arend J, et al. Clinical characteristics and time trends in etiology of hepatocellular cancer in Germany. Digestion. 2013;87(3):147-159.

5. Tacke F, Weiskirchen R. [Liver fibrosis - pathogenesis and novel therapeutic approaches]. Internist (Berl). 2010;51(1):21-29.

6. Gesundheitsbericht fur Deutschland 1998: Kapitel 5.22. http://www.gbe-bund.de/gbe10. Accessed June 5, 2015.

7. Xu J, Murphy SL, Kochanek KD, Bastian BA. Deaths: Final Data for 2013. Natl Vital Stat Rep. 2016;64(2):1-119.

8. Lencioni R, Kudo M, Ye SL, Bronowicki JP, Chen XP, Dagher L, Furuse J, et al. GIDEON (Global Investigation of therapeutic DEcisions in hepatocellular carcinoma and Of its treatment with sorafeNib): second interim analysis. Int J Clin Pract. 2014;68(5):609-617.

9. Poobalan A, Aucott L. Obesity Among Young Adults in Developing Countries: A Systematic Overview. Curr Obes Rep. 2016;5(1):2-13.

10. Wong RJ, Aguilar M, Cheung R, Perumpail RB, Harrison SA, Younossi ZM, Ahmed A. Nonalcoholic steatohepatitis is the second leading etiology of liver disease among adults awaiting liver transplantation in the United States. Gastroenterology. 2015;148(3):547-555.

11. Gines P, Quintero E, Arroyo V, Teres J, Bruguera M, Rimola A, Caballeria J, et al. Compensated cirrhosis: natural history and prognostic factors. Hepatology. 1987;7(1):122-128.

12. D'Amico G, Garcia-Tsao G, Pagliaro L. Natural history and prognostic indicators of survival in cirrhosis: a systematic review of 118 studies. J Hepatol. 2006;44(1):217231.

13. Sporea I, Sirli R, Popescu A, Danila M. Acoustic Radiation Force Impulse (ARFI)-a new modality for the evaluation of liver fibrosis. Med Ultrason. 2010;12(1):26-31.

14. Martinez SM, Crespo G, Navasa M, Forns X. Noninvasive assessment of liver fibrosis. Hepatology. 2011;53(1):325335 .
15. Gallotti A, D'Onofrio M, Pozzi Mucelli R. Acoustic Radiation Force Impulse (ARFI) technique in ultrasound with Virtual Touch tissue quantification of the upper abdomen. Radiol Med. 2010;115(6):889-897.

16. Palmeri ML, Wang MH, Rouze NC, Abdelmalek MF, Guy CD, Moser B, Diehl AM, et al. Noninvasive evaluation of hepatic fibrosis using acoustic radiation forcebased shear stiffness in patients with nonalcoholic fatty liver disease. J Hepatol. 2011;55(3):666-672.

17. Bamber J, Cosgrove D, Dietrich CF, Fromageau J, Bojunga J, Calliada F, Cantisani V, et al. EFSUMB guidelines and recommendations on the clinical use of ultrasound elastography. Part 1: Basic principles and technology. Ultraschall Med. 2013;34(2):169-184.

18. Sporea I, Bota S, Gradinaru-Tascau O, Sirli R, Popescu A. Comparative study between two point Shear Wave Elastographic techniques: Acoustic Radiation Force Impulse (ARFI) elastography and ElastPQ. Med Ultrason. 2014;16(4):309-314.

19. Friedrich-Rust M, Nierhoff J, Lupsor M, Sporea I, Fierbinteanu-Braticevici C, Strobel D, Takahashi H, et al. Performance of Acoustic Radiation Force Impulse imaging for the staging of liver fibrosis: a pooled meta-analysis. J Viral Hepat. 2012;19(2):e212-219.

20. Saverymuttu SH, Joseph AE, Maxwell JD. Ultrasound scanning in the detection of hepatic fibrosis and steatosis. Br Med J (Clin Res Ed). 1986;292(6512):13-15.

21. Jaffer OS, Lung PF, Bosanac D, Patel VM, Ryan SM, Heneghan MA, Quaglia A, et al. Acoustic radiation force impulse quantification: repeatability of measurements in selected liver segments and influence of age, body mass index and liver capsule-to-box distance. Br J Radiol. 2012;85(1018):e858-863.

22. Goertz RS, Egger C, Neurath MF, Strobel D. Impact of food intake, ultrasound transducer, breathing maneuvers and body position on acoustic radiation force impulse (ARFI) elastometry of the liver. Ultraschall Med. 2012;33(4):380-385.

23. Tsochatzis EA, Gurusamy KS, Ntaoula S, Cholongitas E, Davidson BR, Burroughs AK. Elastography for the diagnosis of severity of fibrosis in chronic liver disease: a meta-analysis of diagnostic accuracy. J Hepatol. 2011;54(4):650-659.

24. Bota S, Sporea I, Sirli R, Popescu A, Danila M, Sendroiu $M$. Factors that influence the correlation of acoustic radiation force impulse (ARFI), elastography with liver fibrosis. Med Ultrason. 2011;13(2):135-140.

25. Motosugi U, Ichikawa T, Niitsuma Y, Araki T. Acoustic radiation force impulse elastography of the liver: can fat deposition in the liver affect the measurement of liver stiffness? Jpn J Radiol. 2011;29(9):639-643. 